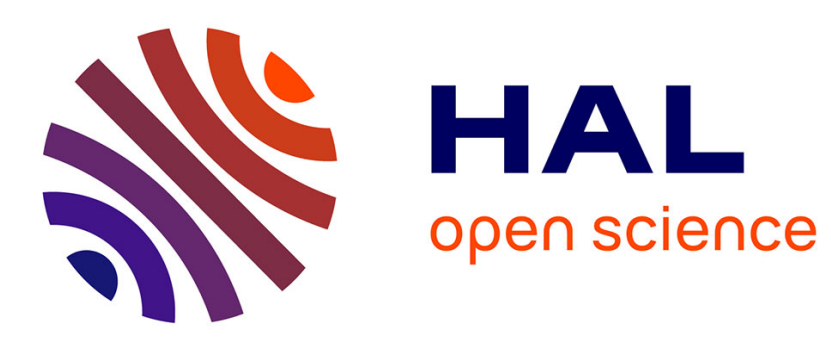

\title{
High-Order Hybrid RANS/LES Strategy for Industrial Applications
}

G. Pont, P. Brenner, Paola Cinnella, Jean-Christophe Robinet

\section{To cite this version:}

G. Pont, P. Brenner, Paola Cinnella, Jean-Christophe Robinet. High-Order Hybrid RANS/LES Strategy for Industrial Applications. Direct and Large-Eddy Simulation X, 24, Springer, pp.313-319, 2017, ERCOFTAC Series book series, 9783319632124. 10.1007/978-3-319-63212-4_39 . hal-02568474

\section{HAL Id: hal-02568474 https://hal.science/hal-02568474}

Submitted on 9 May 2020

HAL is a multi-disciplinary open access archive for the deposit and dissemination of scientific research documents, whether they are published or not. The documents may come from teaching and research institutions in France or abroad, or from public or private research centers.
L'archive ouverte pluridisciplinaire HAL, est destinée au dépôt et à la diffusion de documents scientifiques de niveau recherche, publiés ou non, émanant des établissements d'enseignement et de recherche français ou étrangers, des laboratoires publics ou privés. 


\title{
High-order hybrid RANS/LES strategy for industrial applications
}

\author{
G. Pont, P. Brenner, P. Cinnella, and J.-C. Robinet
}

\section{Introduction}

Turbulent flows of industrial interest are often dominated by large turbulent structures. A typical example is provided by launcher base flows, combining one or more extended and interacting separated regions with strong compressibility effects. Such flow features represent a challenge for CFD simulations, since, on the one hand, well established Reynolds-Averaged-Navier-Stokes (RANS) solvers cannot represent such highly unsteady and three-dimensional large scales and, on the other hand, Large Eddy Simulation (LES) approaches remain too expensive for routine production use in industry. In fact, LES methods can lead to inaccurate results if the mesh size and/or the scheme accuracy are not good enough to resolve correctly most of the relevant flow scales. In complex industrial applications, the energy spectrum is often ill defined and changes from one point to another of the simulation, so that it is difficult to warrant a sufficient resolution everywhere, unless extremely fine meshes are used. Mesh resolution requirements become particularly severe if industrial codes based on low order, low resolution discretization schemes are used. In this work we assess a recently developed hybrid RANS/LES strategy, combining a self-adaptive hybrid turbulence model [Perot et al, 2007] and a hybrid high-order finite volume scheme [Pont et al., 2013], for flow around industrial geometries, namely, the Ariane 6 and Ariane 5 space launchers. Increasingly complex geometrical details are included in the simulation and the results are compared with the experimental data available for the average and root mean square (rms) of the longitudinal distribu-

G. Pont · P. Brenner

Airbus Defense \& Space, Les Mureaux, France e-mail: \{gregoire.pont, pierre. brenner\} dastrium. eads. net

P. Cinnella $\cdot$ J.-C. Robinet

Laboratoire DynFluid, Arts et Métiers ParisTech, Paris, France e-mail: \{paola.cinnella, jean-christophe. robinet \} densam.eu 
tion of the pressure coefficient, which represent a significant quantity of interest for launcher design.

\section{Numerical methodology}

The simulations are carried out by using a high-order unstructured finite volume solver. The space discretization scheme uses $k$-exact reconstructions of the variables over each cell to obtain high-order approximations of the fluxes at cell interfaces. Precisely, the reconstruction process requires approximations of the solution derivatives up to order two, leading to a third-order accurate approximation on generic grids. Derivative approximations are obtained by means of an efficient successive reconstruction technique [Haider et al., 2011]. An exact Riemann solver is then applied to the reconstructed solution at cell interfaces. The resulting numerical scheme is suitable for highly compressible flows since it has good shock capturing capabilities. Nevertheless, it appears to be too dissipative for scale-resolving simulations since it overdamps the vortical structures associated to LES regions of the computational domain. To overcome this difficulty, a hybrid numerical scheme is constructed by locally recentering the third-order scheme in vortex-dominated regions according to a blending function involving Ducros' compressibility/vorticity sensor [Ducros et al., 1999] and a stability criterion involving the grid Reynolds number based on the sum of the local molecular and turbulent viscosity. The fully centered scheme is fourth-order accurate. If the stability criterion is not satisfied, the blending function partly re-centers the scheme while keeping a minimal amount of numerical dissipation to ensure stability. The proposed scheme is referred-to as vortex-centered (VC) scheme. More details can be found in Ref. [Pont et al., 2013]. The proposed strategy has been implemented within FLUSEPA, the unstructured finite-volume solver developed by Airbus Defence \& Space (ADS) to calculate compressible, multidimensional, unsteady, viscous and reactive flow over bodies in relative motion. Time integration is carried out by means of the explicit second-order accurate Heun scheme, along with a time-consistent local time stepping technique.

Unresolved turbulence effects on the resolved scales are taken into account by means of a self-adaptive hybrid RANS/LES model, initially developed by Perot and Gadebusch [Perot et al, 2007], based on a modification of the well known $k-\varepsilon$ model. The model is referred-to as PG hereafter. Such a model acts as a subgrid model in well-resolved regions while keeping a classical $k-\varepsilon$ model in underresolved regions, via a sensor based of the local amount of resolved kinetic energy compared to the modeled one. When the amount of resolved kinetic energy is low compared to an indicator of the local grid resolution, the model switches automatically from the RANS to the LES mode. The original model was developed for free turbulence. In order to apply the model to wall-bounded flows, we introduce a shielding function near the wall, similar to that introduced in [Spalart, 2009]. Wall functions are used near the wall [Launder and Spalding, 1974] to avoid too severe restrictions on the maximum allowable time step. 
The proposed numerical methodology was assessed against well documented test cases [Pont et al., 2013, Pont et al., 2014]. In particular, simulations of the flow around 2D and axi-symmetric backward facing steps showed that the proposed approach provides satisfactory results by using relatively coarse computational grids compared to similar calculations available in the litterature.
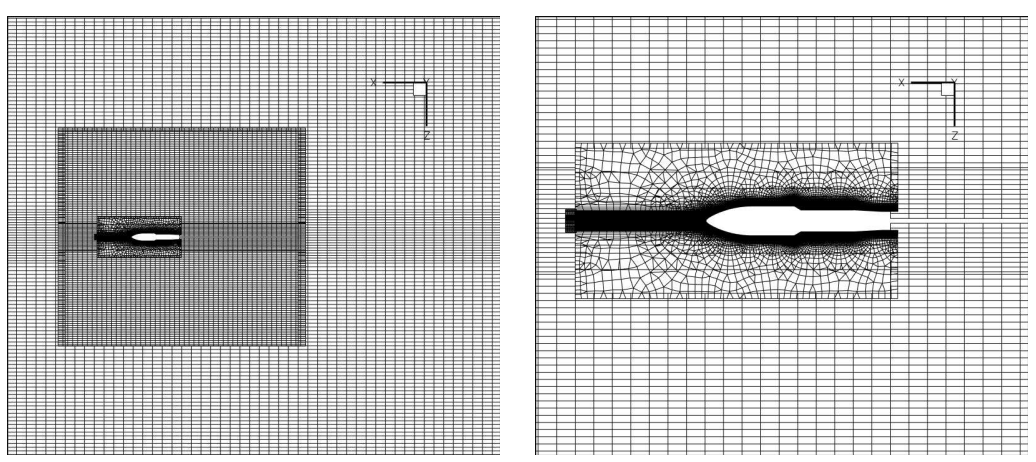

Fig. 1 Details of the overlapping grid around the Ariane 6 shrinkage.

\section{Application to the RANS/LES simulation of industrial launcher configurations}

\subsection{Flow around the shrinkage of Ariane 6}

We simulate a 1/60 scale of the wind tunnel model of Ariane 6 space launcher, with an angle of attack and yaw angle equal to 0 . Simulations are carried out for a Reynolds number, based on the main diameter $D$ of the launcher equal to $9.1 \times 10^{5}$ and a Mach number equal to 0.9. Figure 1 shows the composite grids used to discretize the computational domain. The total number of cells is about $15 \times 10^{6}$. The mesh resolution in the wall-normal and longitudinal directions is of approximately 30 and 50 wall units, respectively. An azimuthal step of 2 degrees is used. The calculations are started from a partially converged $k-\varepsilon$ solution. Statistics are collected over a non-dimensional duration $T \times U_{\infty} /(D-d)=200$ (with $U_{\infty}$ the freestream velocity and $D$ and $d$ the diameters upstream and downstream of the shrinkage). Figure 2 (a) shows the instantaneous Q criterion around the skinkage of the launcher colored by Mach number. In the recirculation area, the heads of Kelvin-Helmholtz vortices are supersonic and induce local compressibility effects. A shock/boundary layer interaction occurs on the fairing of the launcher. The proposed numerical approach accurately captures Kelvin-Helmoltz instabilities while ensuring robustness. The present results are compared to the experiments available for a slightly different configuration [Kumar, 2002]. In the mean flow, see figure 3 (a), the predicted reattachment length, normalized with shrinkage height $(h=(D-d) / 2)$, is equal to 6.1, the experimental value being 5.8. Finally, figure 3 (b) shows that the kinetic 
energy is fully modeled in the attached upstream boundary layer, while in the shear layers and recirculation bubble the modeled kinetic energy is gradually replaced by the resolved one. Figures (a) and (b) shows respectively the evolution of mean pressure and rms pressure coefficients along the shrinkage, versus the longitudinal coordinate normalized by the recirculation length. Both are in good agreement with the experiments. The Strouhal number based on the buffeting frequency, the recirculation length $x_{r}$ and $U_{\infty}$ is about 0.63 , a value close to the experiments and other calculations in the litterature.

Fig. 2 Instantaneous $Q$ criterion colored by the Mach number.

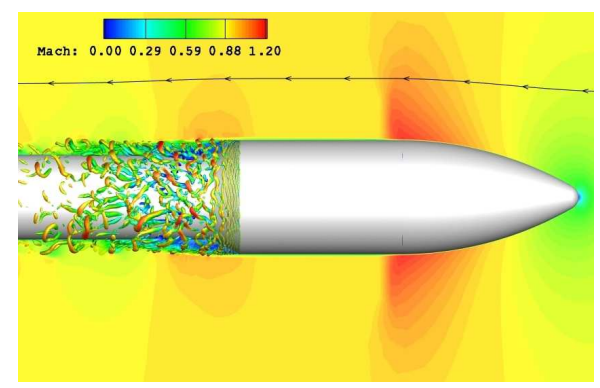

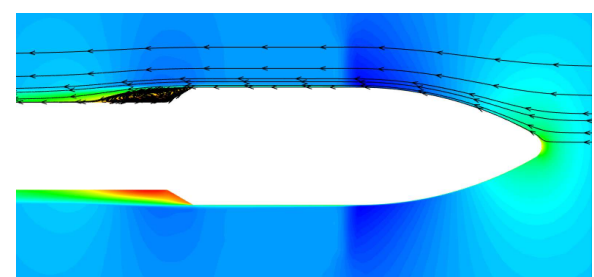

(a)

Fig. 3 Mean flow around the Ariane 6 launcher: a) mean longitudinal velocity and streamlines, b) modeled to total kinetic energy ratio.
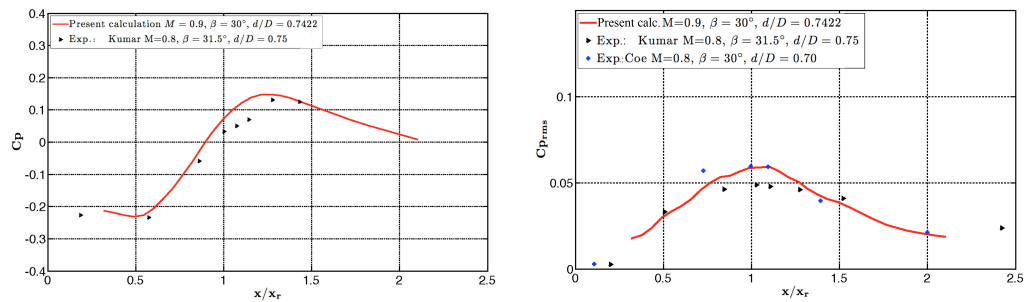

Fig. 4 Distributions of the average (left) and rms (right) pressure coefficient along the shrinkage.

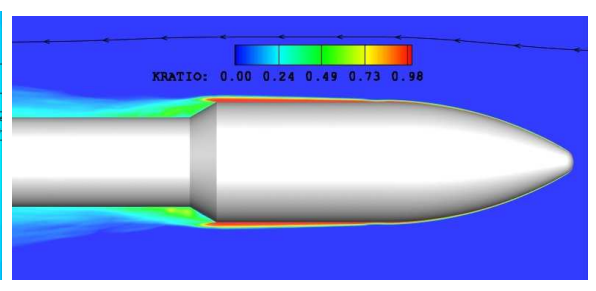

(b) 


\subsection{Flow around the Ariane 5 launcher with boosters}

The next application is a 1/60 scale model of the Ariane 5 launcher at zero angle of attack. The freestram Mach number is equal to 0.8 and the Reynolds number based on the diameter of the central body is $2 \times 10^{-6}$. The computational mesh, shown in fig. 5 is composed by $20 \times 10^{6}$ cells. The $y^{+}$is about 20 at the beginning of the separated region. The azimuthal resolution equal to 2 degrees. The simulation is initialized with a partly converged RANS solution, and the statistics are collected over a non-dimentional duration $T * U_{\infty} / H$ equal to 40 after the numerical transient. This is not long enough to fully converge the statistics, so that results heareafter are to be taken with caution. Figure 6 gives an overview of the instantaneous field at the base of the launcher. The Kelvin-Helmholtz instabilities are clearly visible and are triggered without delay. Figure 7 compares present results for the average and rms pressure coefficient with experimental data collected at ADS the recirculation area of the launcher. The overall agreement is reasonably good.
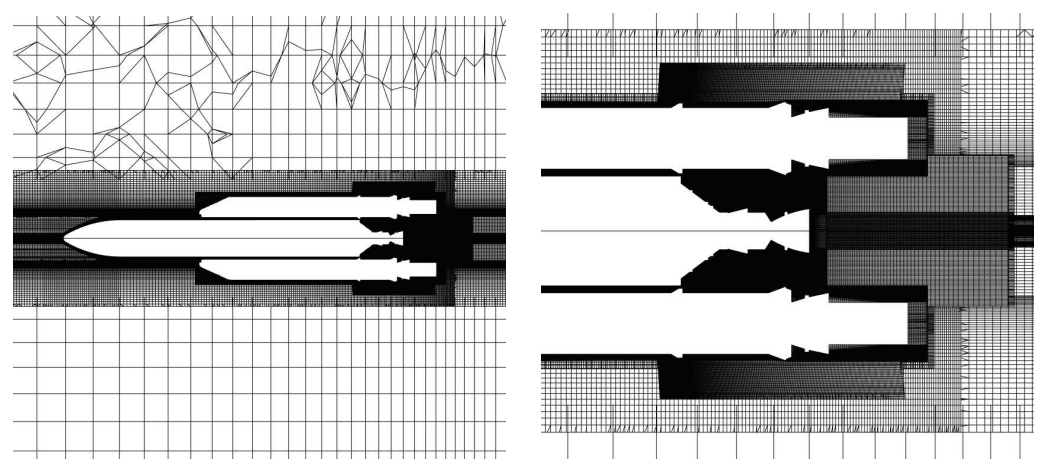

Fig. 5 Details of the overlapping grid around the Ariane 5 launcher.

Fig. 6 Instantaneous Q criterion colored by the Mach number.

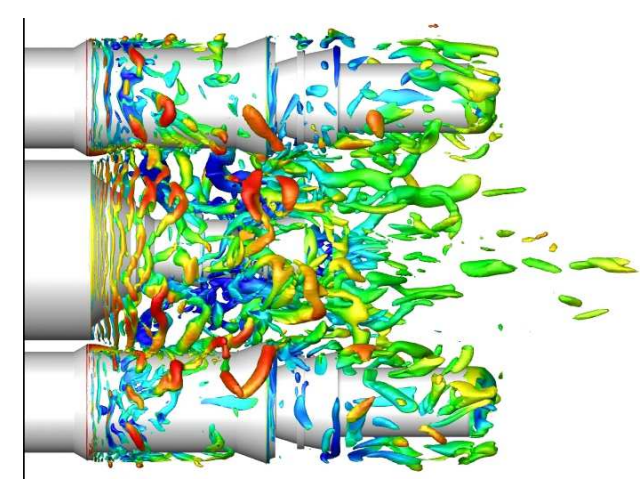




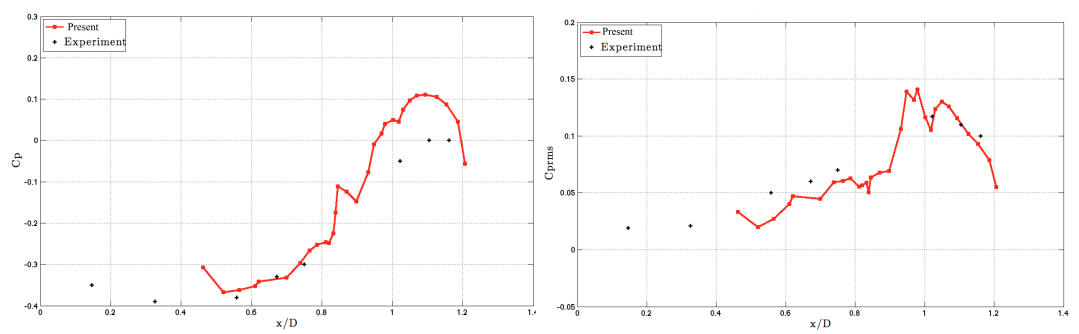

Fig. 7 Distributions of the average (left) and rms (right) pressure coefficient along the nozzle.

\section{Conclusions}

The present work demonstrated the capability of a hybrid high-accurate industrial solver to successfully predict complex launcher configurations to within engineering accuracy, while ensuring the necessary robustness. Using a low-dissipative scheme in conjunction with a hybrid RANS/LES model is found to be crucial for triggering flow instabilities and obtaining correct average and rms wall pressure levels.

\section{References}

[Chauvet, 2007] Chauvet, N. : Simulation numérique et analyse physique d'un jet propulsif contrôlé par des injections radiales. PhD Thesis, University of Poitiers (2007).

[Ducros et al., 1999] Ducros, F., et al.: Large eddy simulation of the shock/turbulence interaction. J. Comput. Phys., 152 517-549 (1999).

[Haider et al., 2011] Haider, F., Brenner, P., Courbet, B., Croisille, J. P.: Efficient implementation of high order reconstruction in finite volume methods. Finite Volumes for Complex Application VI-Problem \& Perspectives, Springer Proceedings in Mathematics, 4 553-560 (2011).

[Kumar, 2002] Kumar, R., Viswanath, P.R. : Mean and Fluctuating Pressure in Boat-Tail Separated Flows at Transonic Speeds. J Spacecraft Rockets, 19(3), 430-438 (2002).

[Launder and Spalding, 1974] Launder, B., Spalding, D. : Application of the energy-dissipation model of turbulence to the calculation of flow near a spinning disc. Letters Heat Mass Transfer, 1, 131-138 (1974).

[Perot et al, 2007] Perot, B., Gadebush, J : A self-adapting turbulence model for flow simulation at any mesh resolution. Phys. Fluids, 19, 1-11 (2007).

[Pont et al., 2013] Pont, G., Cinnella, P., Brenner, P. , Robinet, J-C.: Development of Numerical Schemes for Hybrid Turbulence Modelling in an Industrial CFD Solver, AIAA Paper 20132440.

[Pont et al., 2014] Pont, G., Cinnella, P., Brenner, P. , Robinet, J-C.: Assessment of automatic Hybrid RANS/LES Models for Industrial CFD. AIAA Paper 2014-2691.

[Pont et al., 2014] Pont, G., Cinnella, P., Brenner, P., Robinet, J-C.: An efficient k-exact reconstruction method for robust and high-order finite volumes on any grid. Submitted (2015).

[Spalart, 2009] Spalart, P. R.. Detached eddy simulation. Ann. Rev. Fluid Mech., 41:181202, 2009. 